\title{
Clinico-Epidemiological Profile of Pemphigus: An Observational Study
}

\author{
Authors \\ Anand J Asia ${ }^{1}$, Rachana A Laul ${ }^{2}$ \\ ${ }^{1} \mathrm{MD}$, Professor, Dept of Dermatology, Venereology and Leprosy, Government Medical College Akola \\ ${ }^{2} \mathrm{MD}$, Associate Professor, Dept of Dermatology, Venereology and Leprosy, Govt Medical College Akola \\ Corresponding Author
}

Anand J Asia

Flat no. 303, Sri Sai Gajanan Residency, Bhagwatwadi, Akola, Maharashra, India

Email: anjuasia2010@gmail.com, Phone 9822360284

\begin{abstract}
Background: Pemphigus is a group of rare, chronic, autoimmune, potentially fatal vesiculobullous diseases of the mucous membranes and skin. Clinical profile and epidemiologic characteristics varies in different parts of the world. Aim: The aim of this study was to evaluate the clinical and epidemiological features of pemphigus in our hospital and to compare the results with those in the literature.

Methods: All newly registered patients with confirmed pemphigus admitted at the Department of Dermatology between the period August 2009 and July 2015 were evaluated. Demographic variables and clinical presentation, treatments received were recorded.

Results: Out of 91 patients diagnosed as pemphigus in our centre during the period of 6 years pemphigus vulgaris was the most common type (91.2\%). The mean age of onset was 45 years. Male to female ratio in the patients with pemphigus vulgaris was 1: 1.5. Oral lesions were found in $84 \%$ of patients and it was the first manifestation in 66\%. Mortality rate was $1 \%$ (2 cases) which was due to sepsis. There was no mucosal involvement in patients with pemphigus foliaceus. Only 1 patient had a positive family history. No seasonal predominance for the onset of the disease was noted.

Conclusion: Pemphius vulgaris is the predominant form of pemphigus seen followed by pemphigus foliaceus. Oral mucosa as well as skin are the major sites of involvement. Due to increase in life expentancy occurance of Pemphigis is not an uncommon condition in elderly individuals.

Keywords- Pemphigus vulgaris, pemphigus foliaceus, clinico-epidemiology, skin, oral
\end{abstract}

\section{INTRODUCTION}

Pemphigus is a life threatening autoimmune chronic bullous disease that involves the squamous epithelia and mucous membranes, manifested as painful erosions, loose blisters, and ulceration of skin and mucous membrane. It affects 0.1-0.5/ 100.000 patients per year. The common types include Pemphigus vulgaris (PV), its variants Pemphigus vegetants (P Veg) and Pemphigus foliaceus (PF) with its variant Pemphigus herpetiformis and Pemphigus Erythematosus (PE). Induced Pemphigus, IgA Pemphigus, and paraneoplsatic Pemphigus ${ }^{(1)}$.

Pemphigus vulgaris (PV) is the most common type accounting for approximately $80 \%$ of cases worldwide. It is the commonest autoimmune 
blistering disease in Eastern countries, such as India, malasia, China and middle East. Women are more likely to be affected than men and mean age of onset is approximately 4-6 decades. It is infrequent in children and adolescents but some cases have been reported. The major sites of involvement are oral mucosa and skin. It has been observed more frequently in certain people, e.g., Ashkenazi Jews, Mediterranean populations and Asians (especially Indians and Japanese), who show some genetic predisposition $^{(2)}$. A relationship has been found with HLA, especially with certain HLA class II alleles. Additionally the genetic background, environmental factors, such as drugs, foods, chemicals, viruses, physical agents and stress, have been admitted as triggers of pemphigus.

Pemhigus foliaceus (PF) is less common than PV in most parts of the world and accounts for only 10-20\% of cases of pemphigus. Endemic pemphigus foliaceus is common in rural parts of south America particularly certain states of Brazil (where it is known as fogo salvem) and in Tunisia. PF is noticed more than PV in Mali, Libiya and south Africa where it predominantly affects black races. Pemphigus vegetants a rare variant of Pemphigus vulgaris is characterized by vegetating erosions, primarily in flexures. Oral cavity is almost invariabily involved ${ }^{(3)}$. Pemphigus herpetiformis is a rare variant of Pemphigus foliaceus which resembles Dermatitis herpetiformis in early stages. Pemphigus Erythematosus (PE) is a rare variant of Pemphigus foliaceus having immunological features of both lupus erythematosus and pemphigus. Induced Pemphigus occurs due to drugs which may induce or exacerbate pemphigus . A wide variety of drugs have been implicated such as thiol drugs (captopril, penicillamine, enalapril) and non-thiol drugs (cephalosporins, penicillins, piroxicam nifidepine, chloroquine. hydroxychloroquine). IgA Pemphigus is characterized by intercellular $\operatorname{IgA}$ deposition in the epidermis. Paraneoplsatic Pemphigus has been described with lymph proliferative disorders and sometimes with thymoma, sarcomas and carcinomas. Clinical features overlap with erythema multiforme and lichen planus pemphigoides.
This observational non analytical study was undertaken in patients attending Department of Dermatology to evaluate the prevalence of Pemphigus vulgaris and clinico epidemiological factors associated with it.

\section{MATERIAL AND METHODS}

The data was collected from in-patient record book in the Department of Dermatology in a tertiary care hospital in India for a period of 6 years (August 2015 to July 2015).

The files of all the patients were collected and the data were recorded. Clinical variant of disease, gender, occupation, age at the onset of disease, family history, geographic location of residence, symptoms and clinical presentation, were documented in all cases. The involvement of other mucosal membranes and/or skin was also documented. The severity of skin involvement was categorized as mild with less than 20 vesicles/erosions, moderate with $20-40$ vesicles/ erosions and severe in those with above 40 vesicles/erosions. Mucosal involvement was categorized as mild with 1- 5 lesions, moderate with 5- 10 lesions and severe with lesions above 10 or extensive lesions. Tzanks smear and histopathology examination with or without direct immunofluorescence was the method of confirmation of the diagnosis in all cases.

\section{RESULTS}

During the study period total 91 cases of Pemphigus were diagnosed as having pemphigus . Among these pemphigus vulgaris was the most predominant type of pemphigus seen $(83,91.2 \%)$. followed by pemphigus foliaceus in $6(6.5 \%)$ patients and Linear IgA pemphigus in $2(2.1 \%)$ patient. Pemphigus erythematosus, drug-induced pemphigus, paraneoplastic and Pemphigus vegetans were not found in any of the patient. 83 newly diagnosed cases of pemphigus vulgaris were evaluated.

The male to female ratio was 1:1.5 (33 men , 50 women). The minimum age of patient with pemphigus vulgaris was found to be 11 and maximum was 80 year with an average age of 45.9 
years. The majority of the cases were in the 45-60 year-age group 27 (32.5\%), followed by 24 (29\%) in age group $31 \mathrm{yrs}-45 \mathrm{yrs}$.

Table 1) :- Age and sex distribution of Pemphigus Vulgaris patients.

\begin{tabular}{|l|rl|cc|cc|}
\hline $\begin{array}{l}\text { AGE } \\
\text { GROUP } \\
\text { (YEARS) }\end{array}$ & \multicolumn{2}{|c|}{ MALE } & FEMALE & \multicolumn{2}{|c|}{$\begin{array}{l}\text { MALE+FEM } \\
\text { ALE }\end{array}$} \\
\hline $0-15$ yrs & 0 & $(0 \%)$ & 1 & $(1.2 \%)$ & 1 & $(1.2 \%)$ \\
\hline 16 yr - 30 yrs & 5 & $(6 \%)$ & 12 & $(14.5 \%)$ & 17 & $(20.4 \%)$ \\
\hline 31 yrs- 45 yrs & $11(13.3 \%)$ & 13 & $(15.7 \%)$ & 24 & $(29 \%)$ \\
\hline 46 yrs- 60 yrs & $12(14.4 \%)$ & 15 & $(18 \%)$ & 27 & $(32.5 \%)$ \\
\hline $\begin{array}{l}61 \text { yrs and } \\
\text { above }\end{array}$ & $05(6 \%)$ & 9 & $(10 \%)$ & 14 & $(16.7 \%)$ \\
\hline & $33(39.7 \%)$ & 50 & $(60.2 \%)$ & 83 & $(100 \%)$ \\
\hline
\end{tabular}

$27(32.5 \%)$ patients presented in first quarter of the year, $23(27.7 \%)$ patients presented in second quarter of the year, $25(30 \%)$ patients presented in third quarter of the year, $18(21.6 \%)$ patients presented in last quarter of the year. Among them, $13 \%$ of the patients were government employees; $17.9 \%$, workers; $15.5 \%$, farmers and $59.07 \%$, housewives. $26(31 \%)$ lived in urban areas and 57 $(69 \%)$ in rural areas. In our case series of 83 , pemphigus vulgaris began with localized lesions (73.3\%) rather than generalized lesions (26.7\%). 23 $(27.7 \%)$ of patients had mild, $36(43.3 \%)$ had moderate and 24 (28.9\%) of patients had severe form of skin disease.

Table 2:- Severity of skin disease and area of involvement in Pemphigus Vulgaris (PV) patients.

\begin{tabular}{|c|l|l|l|l|}
\hline & Mild & Moderate & Severe & Total \\
\hline Skin only & $05(6 \%)$ & $6(7.2)$ & $2(2.4)$ & $13(16 \%)$ \\
\hline $\begin{array}{c}\text { Oral and } \\
\text { skin }\end{array}$ & $13(15.6)$ & $30(36.1)$ & $22(26.5)$ & $65(78 \%)$ \\
\hline
\end{tabular}

In $70(84 \%)$ cases, the oral cavity was involvement sooner or later. $13(16 \%)$ had only cutaneous involvement, 5 (6\%) had only oral involvement and $65(78 \%)$ had both cutaneous and oral involvement $95 \%$ of the patients with oral involvement presented with erosions or ulcers with pain and burning sensation. Conjuctival mucosa was affected in only one patient. The duration of oral lesions was taken as the period between the time when patient had first noticed lesion and attended to our clinic.
The mean duration of pemphigus was 1 month to 13 months. Among 70 patients with oral lesions 26 $(37.1 \%)$ of patients had mild, $23(32.8 \%)$ of patients had moderate and $21(30 \%)$ patients had severe form of oral disease.

Table 3) :- Age and severity of oral involvement in Pemphigus Vulgaris patients.

\begin{tabular}{|l|c|c|c|c|}
\hline $\begin{array}{l}\text { AGE } \\
\text { GROUP } \\
\text { YEARS) }\end{array}$ & Mild & Moderate & Severe & \\
\hline $\begin{array}{c}0 \text { - 15 } \\
\text { yrs yr } 30\end{array}$ & 0 & 1 & 0 & 1 \\
\hline $\begin{array}{l}16 \text { yrs } \\
\text { yr yrs- 45 }\end{array}$ & 5 & 7 & 8 & 20 \\
\hline $\begin{array}{l}31 \text { yrs } \\
46 \text { yrs- 60 } \\
\text { yrs }\end{array}$ & 8 & 8 & 7 & 23 \\
\hline $\begin{array}{l}61 \text { yrs and } \\
\text { above }\end{array}$ & 7 & 1 & 3 & 11 \\
\hline
\end{tabular}

Most commonly affected site was buccal mucosa 46 $(55.4 \%)$ followed by gingival $12(14.5 \%)$, tongue $8(9.6 \%)$, palate $8(9.6 \%)$, lips $6(7.2 \%)$ and floor of mouth $3(3.6 \%)$. The age of cases with pemphigus foliaceus ranged from 24 to 65 years with a mean of 44 years. Male to female ratio was 1 (3men, 3 women). Mean interval between the first onset of disease and the first visit was 16 months. There was no correlation between the mean age of patients with pemphigus vulgaris and pemphigus foliaceus.

\section{DISCUSSION}

The average annual incidence of pemphigus ranges from 0.08 cases/100000 individuals in Finland, 0.10.5 in USA 0.17 in France, 0.44 in Macedonia, 0.47 in Bulgaria, 0.93 in Greece, 1.62 in Israel, 0.67 in Shiraz, and 1.6 in Tehran ${ }^{(4)}$. Among the 91 patients seen, pemphigus vulgaris was the most predominant type of pemphigus seen $(83,91.2 \%)$. followed by pemphigus foliaceus in $6(6.5 \%)$ patients and Linear IgA pemphigus in $2(2.1 \%)$ patient. Higher prevalence of pemphigus vulgaris compared with other variants is reported from Shiraz city, Turkey, Saudi Arabia, Bulgaria, France, Greece, India, Bangladesh, Kuwait, Italy and Gilan province $^{(4)}$. On the other hand, pemphigus 
erythematosus is more prevalent in Finland and pemphigus foliaceus is the dominant variant in Brazil, South Africa, Mali, Tunisia ${ }^{(4)}$. In this study the majority of affected patients were aged between 30 and 60 years at the inception, and the condition has been infrequently recorded in individuals younger than 30 . The mean age of onset was 45.9 years previous studies in Saudi Arabia (43.1 year), Mali (46.7 year) and Turkey (43 year) show similar results.

In a study done in Pakistan and Africa the average age of onset was 33.8 and 41 years respectively which is lower than the results of the present study and reference books. Although pemphigus vulgaris mainly affected middle-aged people, it was reported in 3-year old and 89-year-old patients in some references ${ }^{(5)}$. Youngest and eldest patients in our study were of 11 and 80 years. Maximum number of cases were observed between 30-60 years (51\%) surprisingly $14(16.7 \%)$ of patients were above 60 years of age. With regard to our range of patients, we can conclude that pemphigus is expected to occur in patients with all age groups.

Male to female ratio of 1:1.5 was higher than the reports from Tunisia (1:4.1), Mali (1:4), Italy (1:2.2 Greece (1:2.2) and similar to other studies in India $(1.16)^{(6)}$. Only in Saudi Arabia, a greater number of men were affected by this disorder with male to female ratio of 2.2: 1. In northern America, Finland, Malaysia, England, France, Bulgaria and south eastern America morbidity rate was the same in the case of both men and women ${ }^{(6)}$. In total, we observed female dominance in majority of the literature.

In the present study oral mucous membrane was affected in $70(84 \%)$ cases. In $65(78 \%)$ patients oral as well as skin involvement was noted. These results are compatible with other studies and reference books. In the study of Tehran (capital of Iran), skin and mucous membrane involvement was present at a same time in $70 \%$ of patients ${ }^{(7)}$. In the study of Isfahan (center of Iran) mucous membrane involvement was the first sign in $74 \%$ of patients ${ }^{(5)}$. In the study of Rasht (north of Iran), the beginning of the disease was from mouth in $63 \%$ of patients and in $72.4 \%$ of the patients skin and mucous membrane were involved. The mean interval between the onset of mucosal and dermal involvement was less than 5 months in around half of our cases. This interval was 6-9 months in other studies in India and 5-12 months in Croatia ${ }^{(8)}$.

The most commonly affected site was buccal mucosa 46 (55.4\%) followed by gingival 12(14.5\%), tongue $8(9.6 \%)$, palate $8(9.6 \%)$, lips $6(7.2 \%)$ and floor of mouth $3(3.6 \%)$ similar to our study buccal mucosa was the predominant site affected in other studies ${ }^{(8)}$. Since our cases with pemphigus foliaceus had no mucosal involvement, we can state that the presence of mucosal lesions in patients with pemphigus vulgaris have lead to an early visit to medical centers. These findings are supported by a study conducted in Turkey.

The exacerbation of disease in summer is reported from India and South Africa. Hashimoto showed that excessive heat can increase pemphigus vulgaris antigen expression in the epidermis. Further, the study by Tsankov demonstrated that the onset of clinical manifestations predominantly occurred in spring and summer and the heat of the sun rays can provoke the disease ${ }^{(9)}$. However, pemphigus onset was often in the winter in Shiraz city. In our study no significant increase in cases were seen in any of the seasons. Similarly in Gilan province, there was no relationship between the prevalence of the disease and the seasons.

\section{CONCLUSION}

The results of our study demonstrates that pemphigus vulgaris is the most common type of pemphigus seen and it occurs predominantly in females. It is common in the age group between 30 to 60 years. It is not uncommon in elders above 60 years. The most common presentation of the disease was both cutaneous and mucosal involvement and majority of them started with oral lesions. Buccal mucosa is common site of involvement.

\section{REFERENCES}

1. F. Wojnarowska, A Venning. Immunobulbous diseases. In:. DA Burns, SM Breathnachs, NH Cox, CEM Griffiths editors. Rooks Textbook of Dermatology $8^{\text {th }}$ 
edition. Oxford Blackwell Publishing Ltd. 2010:40.1-40.25

2. J Stanley . Pemphigus. In Irwin Freedberg, Arhtur Eisen, Klaus Wolff editors. Fitzpatricks Dermatology in General Medicine $.5^{\text {th }}$ edition New York. McGraw Hill . chapter 60: 654-661

3. A B Martinez, M. Corcuora, Gomez GE. Oral manifestations of pemphigus vulgaris. $\mathrm{J}$ Clin Exp Dermatol Res . Vol 1; issue 2; 1000112.

4. A. Yazdanfar . Epidemiology of pemphigus in Hamedan (West of Iran). A 10 year retrospective study .Int $\mathrm{J}$ of Pharma Biomed Res 2010. 1(4).157-160.

5. A. Ali, Y Aleraza . Pemphigus vulgaris in Iran epidemiology and profile. 2006 ; 5: 69-71.

6. Z. Jari , N. T. Meibodi, Y. Nahidi . Epidemiology of Pemphigus in North East Iran . Indian J of Dermatology 2007 ; Vol.52 : 188-191

7. C. Davatchi ,C. Valikhani, M. Esmail . Pemphigus . Analysis of 1209 cases . Int J Dermatol . 2005 ; 44 : 470-476.

8. G. Karagoz, Bektas K, M. Umar . Evaluation of Pemphigus cases involving oral mucosa. J of Oral Health and Dental Management . Sept 2014 Vol:13 : 3605-608.

9. N. Tsanko ,S. Vassileva ,J. Kamareshev . Epidemiology of Pemphigus in Sofia Bulgaria : A 16 year retrospective study . Int J Dermatol 2006 ; 39:104-108 